\title{
Hole traps in sodium silicate: first-principles calculations of the mobility edge
}

\author{
Nicole Adelstein*, Christopher S. Olson ${ }^{1, *}$, Vincenzo Lordi* \\ Lawrence Livermore National Laboratory, Livermore CA 94550
}

\begin{abstract}
The structure and properties of $\left(\mathrm{Na}_{2} \mathrm{O}\right)_{0.30}\left(\mathrm{SiO}_{2}\right)_{0.70}$ sodium silicate glass are studied by combined ab-initio and classical molecular dynamics simulations to identify the sources of electronic traps in the band gap. Structures from classical molecular dynamics melt-quench simulations are taken as initial configurations for first-principles density functional theory structural relaxation, from which electronic properties are determined. An ensemble of thirty glass structures, each containing 660 atoms, is prepared in order to perform statistical analyses. The inverse participation ratio is used as a metric to characterize localized states in the band gap and determine the mobility edge. Structures with varying amounts of local disorder (traps) are compared. The most localized and highest energy trap states are due to Si atoms with 2-3 non-bridging oxygen atoms. Control of the electronic properties of amorphous insulators and semiconductors is essential for the advancement of many technologies, such as photovoltaics and scintillators, for which the present analysis is indispensable.
\end{abstract}

Keywords: mobility edge, DFT, sodium silicate, Non-bridging oxygen

\section{Introduction}

The ability to improve or control the electronic conductivity of amorphous insulators will lead to significant advances in many technologies, such as scintillating materials, electrochemical cells, and photovoltaics. First-principles calculations of crystalline electronic structures has enabled the technological

*adelstein1@llnl.gov

${ }^{1}$ North Dakota State University

Preprint submitted to Journal of Non-Crystalline Solids

August 21, 2015

(C) 2015. This manuscript version is made available under the Elsevier user license http://www.elsevier.com/open-access/userlicense/1.0/ 
development of many materials, such as silicon[1], but similar advances in glasses and amorphous materials have been frustrated by the significantly larger computational demands of simulating amorphous materials.

The properties of both amorphous and crystalline materials can be controlled by engineering their local structure and defects. Electronic conductivity is sensitive to substitutional atoms or vacancies that leave dangling bonds, which can donate electronic carriers or trap them. The electronic structure calculations presented in this paper relate the local structure of dangling bonds in sodium silicate glass to electronic traps in and near the band gap. Using first-principles methods to simulate amorphous materials requires judicious selection of an adequately large cell size and simulation length; state of the art supercomputers are making this task possible[2, 3]. Running simulations in parallel on supercomputers also allows statistics to be gathered from an ensemble of structures, as is done for sodium silicate in this study.

Low electronic conductivities in amorphous insulators are not only due to large effective masses, as in wide gap crystalline insulators, but also to carrier traps[4]. We calculate the mobility edge and nature of charge traps in a prototypical amorphous material sodium silicate, which is representative of the alkali-silicates. Alkali and alkali-earth cations are silica network modifiers that create non-bridging oxygen atoms (NBOs) and serve a wide variety of technological applications, such as reducing crystallization[5] and decreasing the glass-transition temperature and water solubility[6]. Compared to silica, which has fewer NBOs, these dangling bonds affect the mobility gap and edge, which distinguishes between delocalized versus localized electronic bands that accommodate band like conductivity versus trap charge, respectively. Cedoped silica is a candidate material for scintillators, but the low mobility of electrons and holes and the low solubility of Ce-dopants means the charge carriers do not often diffuse to the Ce-activator dopans, which has limited its technological use[7].

Theoretical models of electronic conductivity in amorphous insulators started with the seminal paper by Mott in 1977[8]. By 1980, Mott's theories of electron traps and hopping (as contrasted with band-like conductivity) had been applied to explain optical phenomena in sodium silicate[9]. However, the electronic conductivity is difficult to measure conventionally, especially in the case of alkali-silicates, where ionic conduction by the alkali ion can be significant. Luckily, measurements of the luminescence or scintillation of a material can selectively probe the conductivity of electrons and holes. For 
example, researchers developing scintillating glasses have observed no scintillation in Ce-doped sodium trisilicate compared to Ce-doped silica[10, 11, 7], giving indirect evidence of significantly poorer carrier transport in the sodium silicate host. Radio-scintillation efficiency is a measure of carrier transport, because photogenerated electrons and holes must migrate to the activator dopant Ce atoms for scintillation to occur[12]. However, the microscopic cause of the degraded energy transfer in sodium silicate versus silica remains undetermined, especially since there have been very few first-principles studies of electronic transport in alkali glasses.

A detailed analysis of the electronic structure of sodium silicate from first-principles can reveal the structure-property relationship between defects and electronic mobility. Though the electronic structure of silica is well-established [13, 14], there have been only a few, small (90 atoms [15] and 120 atoms[16]) first-principles studies that compare the effect of the NBOs and other atomic-scale defects on the electronic structure of sodium silicate. In Ref. [15], the electronic density of states (DOS) from two $\left(\mathrm{Na}_{2} \mathrm{O}\right)_{0.20}\left(\mathrm{SiO}_{2}\right)_{0.80}$ configurations were compared with the DOS from silica, but in a way that obscures the trap states that form in the gap. Our analysis of $\left(\mathrm{Na}_{2} \mathrm{O}\right)_{0.30}\left(\mathrm{SiO}_{2}\right)_{0.70}$, which contains a higher concentration of NBOs than in Ref. [15], identifies the atomic geometries of the NBOs in the mobility gap. Our simulation cell was chosen to be large (660 atoms) so that an ensemble of structures would achieve a good statistical representation of the structure in macroscopic volumes.

\section{Simulation Details}

Simulations of the structure of amorphous materials often employ classical potentials to allow the sampling of many statistically independent configurations. In contrast, first-principles (density functional theory-based) molecular dynamics simulations of structures with hundreds of atoms are often limited to only 10's of picoseconds in length, which is too short to collect uncorrelated time samples. Reverse Monte Carlo techniques have also been employed to simulate amorphous structures[17], but the effect of the glass forming procedure cannot be studied with this method. Thus, we use a classical potential to create glass structures by quenching the melt at quench rates between $10^{11} \mathrm{~K} / \mathrm{s}$ and $10^{14} \mathrm{~K} / \mathrm{s}$. We simulate the $30: 70 \mathrm{Na}_{2} \mathrm{O}: \mathrm{SiO}_{2}$ glass (30:70NS) and compare it with lower Na content tetrasilicate glass (20:80NS). The prior study on 20:80NS used a variable charge BKS two-body potential 
and quenched from $3500 \mathrm{~K}$ to $300 \mathrm{~K}$ at a rate of $5 \times 10^{13} \mathrm{~K} / \mathrm{s}$. We use a three-body potential based on a modified Born-Mayer-Huggins (BMH) ionic potential, described in Ref. [18], with recently optimized parameters, ${ }^{2}$ which we implemented in the lammps MD code[19].

The glass forming procedure starts with swapping out $\mathrm{Si}$ and $\mathrm{O}$ atoms for $\mathrm{Na}$ from a $4 \times 4 \times 4 \beta$-cristobalite cell and setting the volume to correspond to the experimental density of $2.466 \mathrm{~g} / \mathrm{cm}^{3}$. Our 30:70NS simulation cells have $154 \mathrm{SiO}_{2}$ formula units and $66 \mathrm{Na}_{2} \mathrm{O}$ formula units. A time step of 1 fs was employed using the Langevin thermostat with a 1.0 ps damping parameter to control temperature.

Initial structures are generated using the classical potential and the following steps. First, the forces on the atoms are minimized and then the temperature is increased from 300 to $8000 \mathrm{~K}$ over $6000 \mathrm{ps}$, as the volume is scaled up $116 \%$ to take into account thermal expansion. The melt is held at $8000 \mathrm{~K}$ for $80 \mathrm{ps}$, after which 30 snapshots are taken every 32,000 steps (32 ps), in order to create an ensemble of independent glass structures. The melt structure from each snapshot is quenched back down to $300 \mathrm{~K}$ over 80,000 steps ( $80 \mathrm{ps}$ ), while rescaling the volume every 8000 steps. This corresponds to a quench rate of $9.6 \times 10^{13} \mathrm{~K} / \mathrm{s}$, although quench rates as slow as $10^{11} \mathrm{~K} / \mathrm{s}$ were tested and compared. We find a glass transition temperature around $3000 \mathrm{~K}$. The 30:70NS structures compare well to other simulations from the literature, as will be described in the Results section. Finally, each snapshot is equilibrated at $300 \mathrm{~K}$ for $80 \mathrm{ps}$ from which structural statistics are collected.

The final room temperature structures from the above procedure using classical MD are not immediately used to calculate the electronic structure, because very large residual forces remain on the atoms. Thus, each structure is further relaxed with density functional theory (DFT) using the PBE exchange-correlation functional $[20,21]$ to reduce the forces on all atoms to less than $0.05 \mathrm{eV} / \AA$. A planewave cutoff of $700 \mathrm{eV}$ is used to ensure accuracy, based on tests of $\mathrm{SiO}_{2}$, with only the $\Gamma$-point used to sample the Brillouin zone. The VASP code $[22,23,24,25]$ and the projector-augmented wave method [26, 27] was used for all DFT calculations.

The localization of each electronic state near the band gap is quantified using the the inverse participation ratio (IPR), which is related to the second

\footnotetext{
${ }^{2}$ S.H. Garofalini, private communication
} 
moment of the charge density. The IPR compares the spatial extent of a given wave function, $\psi_{n}$, to the total probability density, on a scale from 1 to $N$, where 1 is delocalized and $N$ is very localized. Specifically, the IPR is calculated from the band-decomposed partial charge density, $\left|\psi_{n}(\vec{r})\right|^{2}$, via

$$
I\left(\psi_{n}\right)=N \frac{\sum_{i=1}^{N}\left|\psi_{n}\left(\vec{r}_{i}\right)\right|^{4}}{\left[\sum_{i=1}^{N}\left|\psi_{n}\left(\vec{r}_{i}\right)\right|^{2}\right]^{2}},
$$

where $i$ labels the real-space grid points upon which the charge density is computed, $N$ is the total number of grid points (and maximum IPR value), and $n$ is the band index. For the calculations of IPR, the plane wave cut-off was reduced to $400 \mathrm{eV}$ without loss of accuracy.

All computed quantities are averaged over the ensemble of 30 configurations to capture the wide structural variations and range of defects of a bulk glass sample.

\section{Results}

\subsection{Glass Structure}

The structural analysis of our glass model highlights how the addition of sodium breaks up the network structure of silica.

The metrics we calculate also indicate the quality of the simulation procedure and the potentials used. While, in principle, the moskkt reliable structures would be obtained from performing the melt-quench simulations entirely using DFT, computational feasibility dictates that initial structures be generated using melt-quench MD simulations with classical potentials and then relaxed post-quench with DFT. While the DFT relaxation does change the local structure slightly, the classical potential predominantly determines the structural statistics of the generated glass models.

The addition of sodium to silica disrupts the $\mathrm{Si}-\mathrm{O}-\mathrm{Si}$ bonds, and should generate approximately one non-bridging oxygen atom (NBO) per $\mathrm{Na}$ atom, in a $\mathrm{Si}-\mathrm{O}-\mathrm{Na}$ configuration. In our simulations of 30:70NS, there are $34 \pm$ $0.0 \% \mathrm{NBO}$, which is close to the sodium concentration. In Ispas et al.'s calculation of 20:80NS, a NBO concentration of $22 \%$ was found, which is also similar to, but slightly higher than, the concentration of $\mathrm{Na}$ in the system. The non-bridging oxygen atoms are the primary source of trap states in the 
mobility gap and near the valence band maximum (i.e., Urbach tails) [28, 29], and this is discussed in more detail below.

Each Si cation can bond to 0-4 NBO atoms, with increasing NBO being less energetically favorable. The number of bridging oxygen (BO) atoms bonded to a given $\mathrm{Si}$ atom defines its $\mathrm{Q}^{n}$ value, such that a $\mathrm{Si}$ atom with $n=4 \mathrm{BO}$ atoms is defined as a $\mathrm{Q}^{4} \mathrm{Si}$, whereas a $\mathrm{Si}$ atom with $n=1 \mathrm{BO}$ atom is defined as a $\mathrm{Q}^{1} \mathrm{Si}[30]$. Each $\mathrm{NBO}$ is labeled by the $\mathrm{Q}^{n}$ value of the Si to which it is bonded. A subscript will be added to the label to indicate the oxygen and if there are no NBO, these oxygen will be labeled $\mathrm{Q}_{\mathrm{O}}^{4}$.

In addition, each localized NBO can be assigned an energy level by associating it with a band in the electronic structure, using the following steps:

(i) first, the band structure is calculated and NBO's $\mathrm{Q}_{\mathrm{O}}^{n}$ values are assigned;

(ii) then, the partial charge density from each band is projected onto the atomic orbitals of all atoms in the supercell and the oxygen atom with the highest contribution is determined;

(iii) finally, the $\mathrm{Q}_{\mathrm{O}}^{n}$ value of the highest contributing oxygen is assigned to the band.

For localized electronic states, the charge density extends over only a few atoms, usually including the NBO or other defects. We found it useful to assign one $\mathrm{Q}_{\mathrm{O}}^{n}$ value to each electronic state (band), using the procedure outlined above, rather than report the $\mathrm{Q}_{\mathrm{O}}^{n}$ ratio for each band. However, we also include the average $\mathrm{Q}^{n}$ ratio, as we find that this is the most important metric for characterizing the electronic structure. We also analyze and report the ring statistics, pair distribution functions, and bond angle distributions for completeness.

The ratios of $\mathrm{Q}^{n}$ values can be estimated experimentally from nuclear magnetic resonance (NMR) experiments[30] and provide a check on the fidelity of the glass model. To our knowledge there are no published data of $\mathrm{Q}^{n}$ ratios from NMR experiments on 30:70NS glass; however, several studies have been done on compositions with similar Na:Si ratios. For example, Maekawa et al. published a fit of $\mathrm{Q}^{n}$ values for sodium silicate compositions ranging from $20 \%$ to $55 \% \mathrm{Na}_{2} \mathrm{O}$ mole fractions[31]. From an interpolation of their data, the estimated $\mathrm{Q}^{n}$ ratios for $30: 70 \mathrm{NS}$ are given in the first row of Table 1 and the "Experimental" bars in Fig. 1. Our simulation gives $Q^{n}$ values that are noticeably different from those estimated by the experimental fit, but other simulations of 30:70 NS using two body potentials suffer the same discrepancies [32, 33, 34]. As shown in Fig. 1, the distributions of $Q^{n}$ ratios 


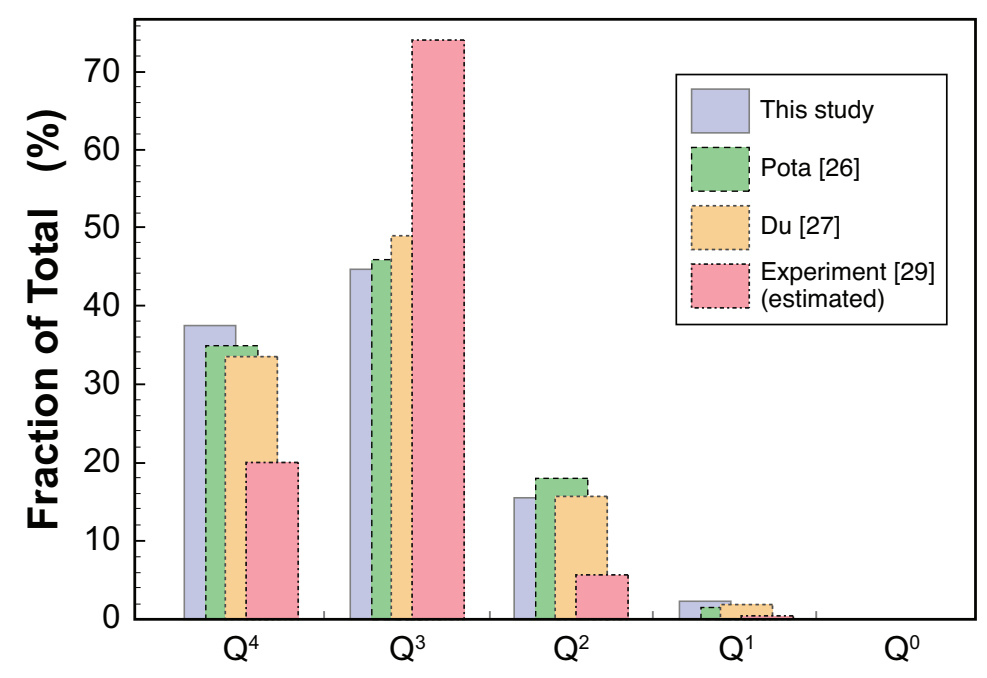

Figure 1: $\mathrm{Q}^{n}$ values from three simulations with different potentials [32, 33], including the one used in this study, are plotted against experiment[35]. The differences between the simulations are small compared to discrepancies with the experiment.

from different simulations are very similar, and all show similar systematic discrepancies with experiment. Also, we note that a previous simulation of 20:80 NS glass (to which we compare our results) also significantly differs from Maekawa et al.'s measured $\mathrm{Q}^{n}$ ratios, with variations over 5 percentage points[15]. In the simulation of the 20:80 NS, two-body potentials with variable charge on the sodium atoms (dependent on distance) were employed to generate the initial glass configurations.

In addition, the radial distribution functions of our model compare well to previous experimental and theoretical reports in the literature. Total correlation functions can be obtained from both neutron diffraction experiments and simulations and used to test the fidelity of generated structures. We report the total correlation function, $T(r)=4 \pi r \rho(r)$ (where $\rho(r)$ is the atom density) in Fig. 2, rather than the radial distribution function, because the function to broaden $T(r)$ that accounts for the neutron scattering length is symmetric and $r$-independent. A comparison of the correlation function between simulation and experiment is quantified using a modified crystallographic $R$ factor[35]:

$$
R_{\chi}=\left(\frac{\sum_{i}\left[T_{e x p}\left(r_{i}\right)-T_{s i m}\left(r_{i}\right)\right]^{2}}{\sum_{i} T_{e x p}^{2}\left(r_{i}\right)}\right)^{\frac{1}{2}}
$$




\begin{tabular}{lcccc}
\hline \hline & $\mathrm{Q}^{4}$ & $\mathrm{Q}^{3}$ & $\mathrm{Q}^{2}$ & $\mathrm{Q}^{1}$ \\
\hline \hline Exp. Est.[31] & 20 & 74 & 5.5 & 0.5 \\
This study MD & 37 & 45 & 16 & 2 \\
This study DFT & 39 & 45 & 14 & 2 \\
Pota [32] & 35 & 46 & 18 & 1 \\
Du [33] & 33 & 49 & 16 & 2 \\
20:80 NS [15] & 56 & 37 & 6 & 0 \\
20:80 NS Exp. [31] & 50 & 48 & 2 & 0 \\
\hline
\end{tabular}

Table 1: The distribution of $\mathrm{Q}^{n}$ ratios for 30:70NS glass estimated from experiment, and compared to simulations using a variety of potentials, including classical and ab initio simulations from this work, as well as 20:80NS glass.

We obtain $R_{\chi}$ of $7.4 \%$ from our classical simulations and $6.7 \%$ from our DFT relaxed simulations, while Yuan and Cormack's simulation yielded $R_{\chi}=$ $5.6 \%$ [36], and Du and Cormack report $R_{\chi}$ of $5.9 \%$ for their structure[33]. Our simulation structure compares slightly less favorably to experiment, which is unsurprising since Yuan and Cormack fit their potential parameters specifically to give low $R_{\chi}$, however all the simulations shows very similar shape and peak positions in the $T(r)$. Both Du and Yuan use partial charge twobody modified Buckingham potentials, while we use a three-body potential to account for the covalent (angle dependent) nature of the $\mathrm{Si}-\mathrm{O}$ bonds. Using different quench rates between $10^{11}$ and $10^{14} \mathrm{~K} / \mathrm{s}$ did not improve the $R_{\chi}$ value significantly, indicating that the potential, rather than the quench rate, is the cause of the discrepancy between the simulated $T(r)$ and the experimental one.

The ring structure is a common metric to describe the mid-range structure of a silicate glass. Unlike for the $\mathrm{Q}^{n}$ ratios and $T(r)$, there are no quantitative experimental probes of the ring structure, but we include our results for completeness, along with our calculation of the bond angle distributions. In quartz, the $\mathrm{SiO}_{4}$ tetrahedra form rings with six $\mathrm{Si}$ alternating with six O. The sodium breaks up the ring structure and in our simulation forms smaller rings. As shown in Fig. 3, our distribution shows more 5 membered rings, which particularly strain the ideal $\mathrm{SiO}_{2}$ bond angle. The ring structure obtained seems dependent on the classical potential employed, as Pota et al. found a maximum in the mean ring distribution of 30:70NS around 6 or 7[32]. We find that a slower quench and anneal step can change the ring distribution slightly, but not in a systematic way. While our ring distribution is different 


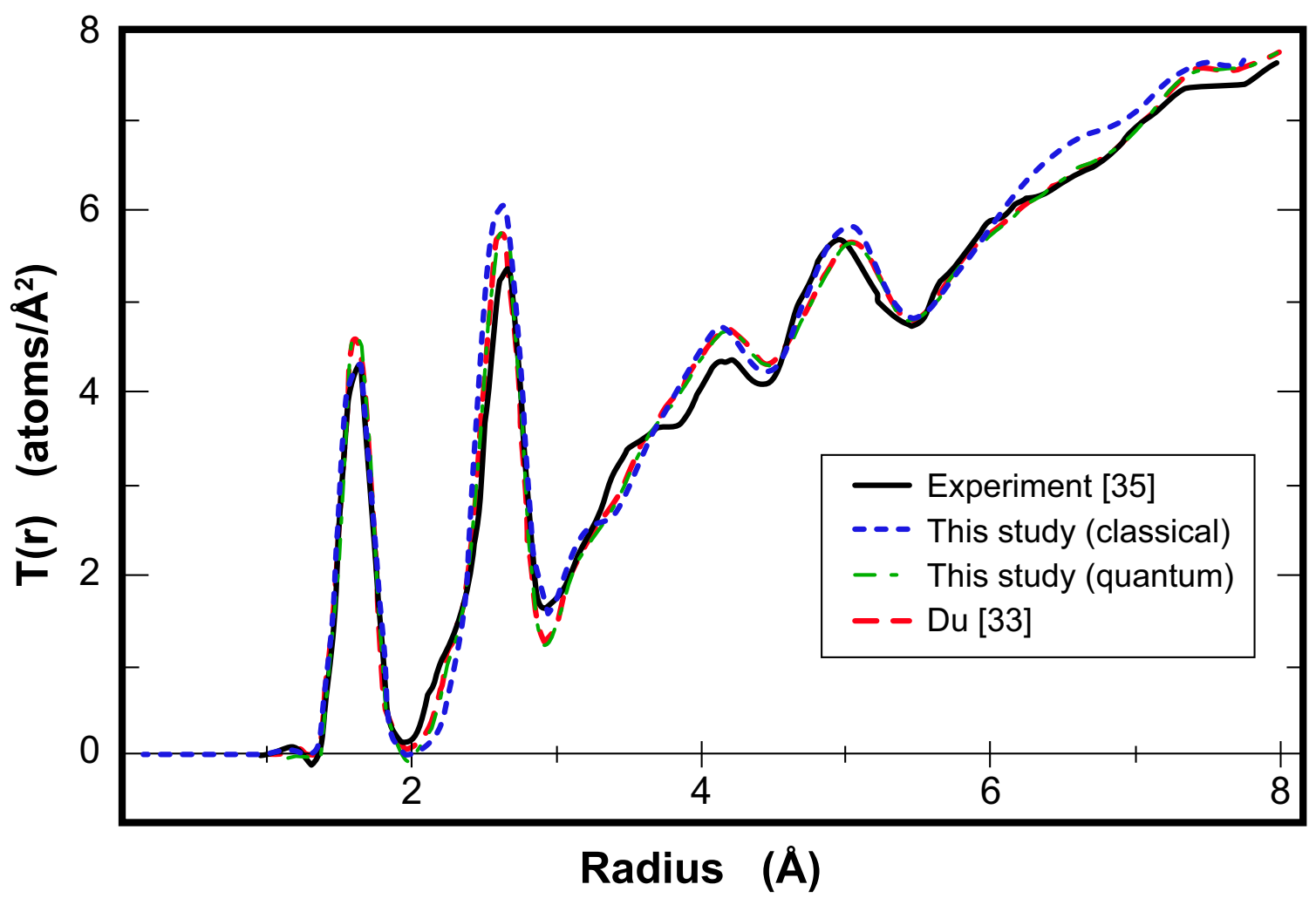

Figure 2: The total correlation function $T(r)$ of $30: 70 \mathrm{NS}$ from this work is compared to experimental neutron diffraction data [35] and a previous classical potential simulation by Du and Cormack [33]. 


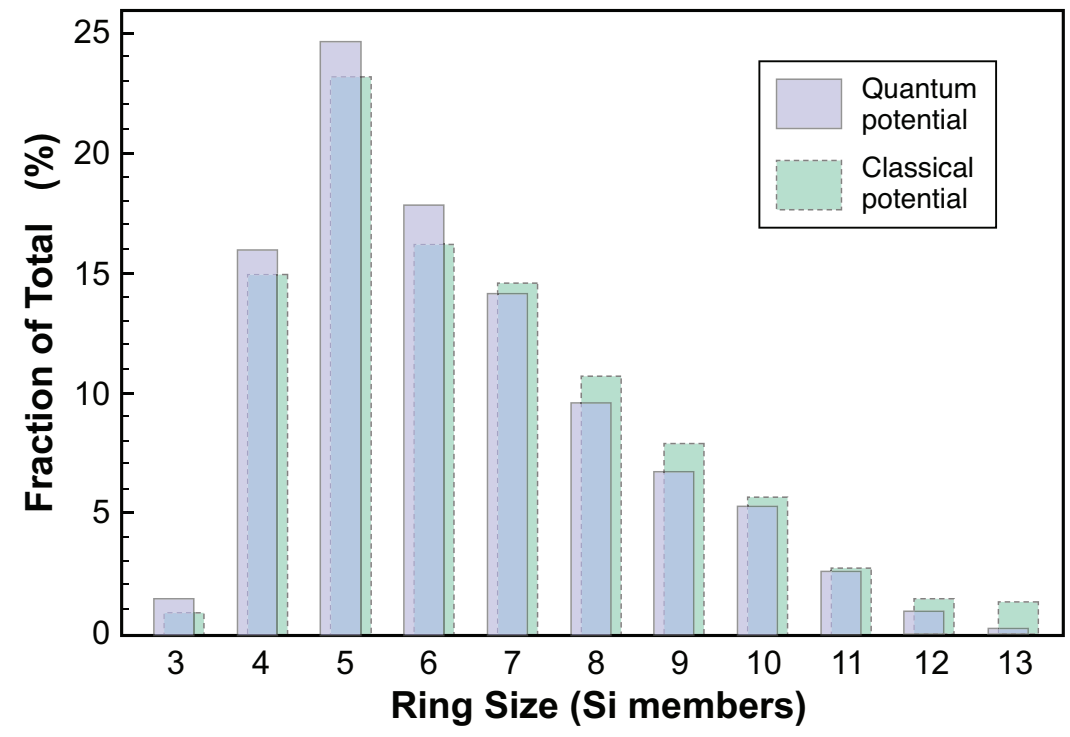

Figure 3: The computed distribution of rings in the 30:70NS glass structure changes slightly after first-principles DFT relaxation.

from Pota et al., the effect on the electronic structure is minimal, since the trap states near the valence band edge are associated with NBO, as we show later; thus, we note that hole traps are probably not associated with strained bonds in rings smaller or larger than $6 \mathrm{Si}$ atoms.

The final glass metric that we report is the bond angle distribution. Our calculated distribution for the $\mathrm{Si}-\mathrm{O}-\mathrm{Si}$ bond angle has a normal distribution with a mean of $153.5^{\circ}$ and a sigma of $12.3^{\circ}$ (full width at half $\max 29.0^{\circ}$ ). The computational results from the literature range between $139^{\circ}$ and $152^{\circ}$ $[33,36,16]$.

In addition to testing the quench rate, we found that simulated annealing at a variety of temperatures between 1000 and $5000 \mathrm{~K}$ does not significantly change the metrics. Ring statistics and the $\mathrm{Q}^{n}$ ratios stay relatively constant with the anneal step. While melted, larger ring sizes are observed and the $\mathrm{Q}^{4}$ ratio increases at the expense of the $\mathrm{Q}^{2}$, since enough thermal energy is available for the tetrahedra to rotate more freely, so non-bridging oxygens favor forming $\mathrm{Si}-\mathrm{O}$ bonds, resulting in more 5 coordinated $\mathrm{Si}$ atoms and a more extended ring structure. But as the system is cooled, the bond angle and ring distributions revert to their quenched values.

With the slowest quench rate of $10^{11} \mathrm{~K} / \mathrm{s}$, the percentage of $\mathrm{Q}^{3}$ decreases 
below the percentage of $\mathrm{Q}^{4}$, while concurrently increasing the percentage of $\mathrm{Q}^{2}$. This relationship between $\mathrm{Q}^{n}$ values is well documented in the literature $[32,33]$ :

$$
\mathrm{Q}^{3} \leftrightarrows \mathrm{Q}^{4}+\mathrm{Q}^{2}
$$

Thus, the slower quench rate results in a $\mathrm{Q}^{n}$ distribution $\left(\mathrm{Q}^{1}=1.9 \%, \mathrm{Q}^{2}=18.4 \%\right.$, $\mathrm{Q}^{3}=36.3 \%, \mathrm{Q}^{4}=42.7 \%$ ) that is further from experiment. As we do not intend to simulate the exact structure of 30:70NS glass (which would require different potentials), but rather understand the effect of increased sodium, and thus increased traps on the electronic structure. Our 30:70 NS structures has more NBO than the 20:80 NS and serves as an adequate comparison.

As noted above, DFT calculates large forces on the structures quenched to $300 \mathrm{~K}$ using the classical potentials, so the structure is further relaxed with DFT. Small quantitative changes in the glass structure metrics result from the DFT relaxation, but the electronic structure does not change significantly. The effect of the DFT relaxation on the structure is subtle. The relaxation does not significantly change the band gap, however, the localized traps near the valence band maximum do change as the number of NBO decreases. The percent of NBO goes from $34 \%$ to $32 \%$ on average for the 30 configurations. The percent of NBO decreases because the number of fivecoordinated Si increases from $2.90 \%$ to $6.75 \%$. Changes in the ring structure and the $\mathrm{Q}^{n}$ ratios are also measurable upon relaxation with DFT. Upon relaxing with DFT, the number of larger rings decreases and the number 5 and 6 Si-membered rings increases, by about 1-2 percentage points. In addition, the number of $\mathrm{Q}^{1}$ and $\mathrm{Q}^{2}$ species decreases (with the decrease of $\mathrm{NBO}$ ) and the number of $\mathrm{Q}^{4}$ increases (with a slight increase in $\mathrm{Q}^{3}$ ), as noted in Fig. 3 and Table 1.

\subsection{Electronic Structure}

The averaged DOS from the 30 quenched configurations after relaxation with DFT-PBE is shown in Fig. 4, comparing 30:70NS, 20:80NS[15], and $0: 100 \mathrm{NS}$ (silica)[15]. We will show that the shoulder at the top of the valence band in the DOS is primarily from localized NBO states; note that this peak is larger in the 30:70NS glass than in the 20:80NS glass, commensurate with the higher concentration of NBO in 30:70NS. The calculated DOS of silica and the tetrasilicate (20:80NS) glass[15] also show a small peak below the conduction band minimum, while the band tail of the conduction band in the 30:70NS is less distinct. While previous calculations have identified the 




Figure 4: The density of states for 30:70 sodium silicate calculated with DFT-PBE and compared to the DFT-PBE DOS of 20:80 sodium silicate and silica from [15].

shoulder of the valence band as due to NBO [29], the following results quantitatively assign the structure and localization of the states in the shoulder.

As seen in Fig. 4, the energy between the highest occupied state and the lowest unoccupied state is about $1.5 \mathrm{eV}$ for $30: 70 \mathrm{NS}$ (within the PBE approximation, which underestimates gaps). However, the mobility gap is significantly larger than that, as many of the states are due to localized NBOs. The mobility gap is defined by the energy difference between the occupied and unoccupied delocalized bands that contribute to band conductivity. While it is difficult to determine the precise positions of the mobility edges in the valence and conduction bands, first-principles calculations of the localization of states can help define the mobility edges, as indicated in Fig. 5, by quantitatively analyzing the delocalization of states. The localization criterion used to assign the mobility edges from the IPR values is described below in the Discussion section.

Figure 5 shows the logarithm of the IPR values of around 1200 valence and conduction band states near the band gap from each of the 30 glass 


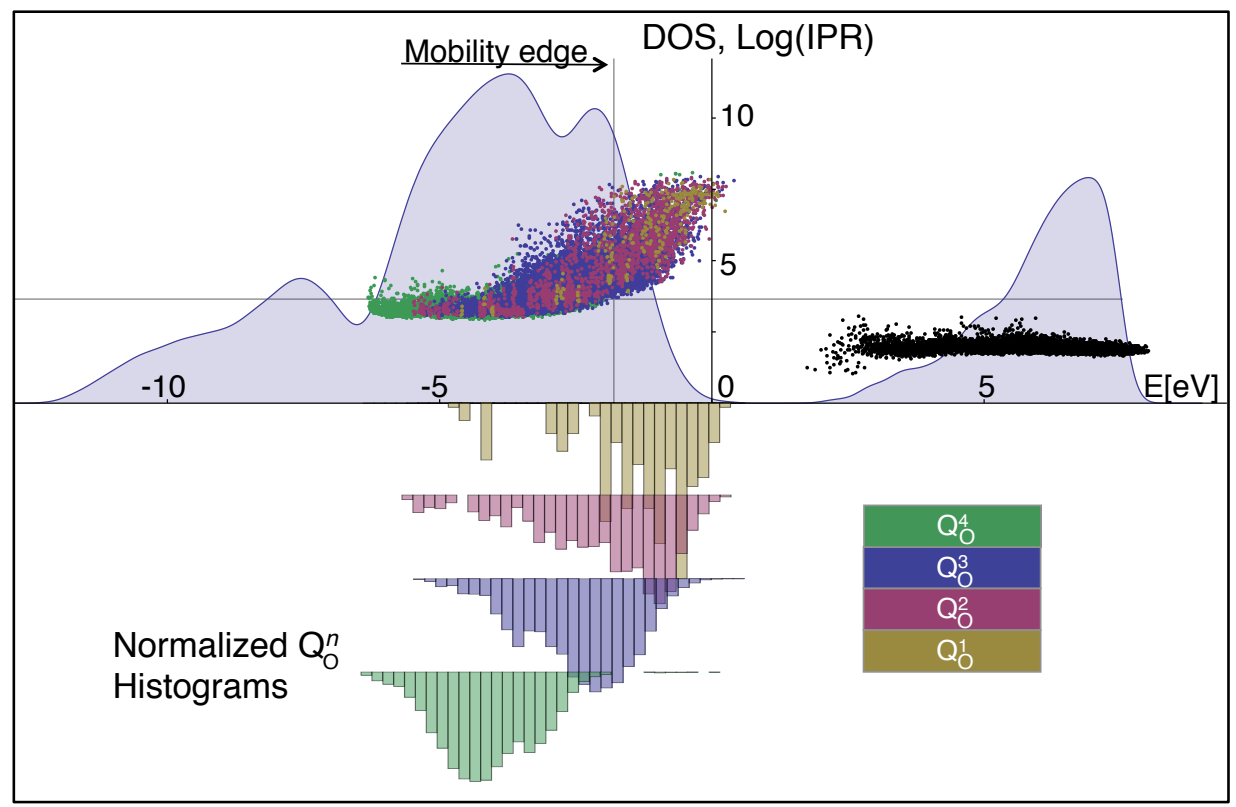

Figure 5: The $\log (\mathrm{IPR})$ for each state around the band gap is plotted over the total DOS, with guides to the eye to indicate the mobility edge. The color of each point corresponds to the $\mathrm{Q}_{\mathrm{O}}^{n}$ assignment of each state, according to the color key at the bottom-right. Below the DOS, normalized histograms of the energy states indicate the $\mathrm{Q}_{\mathrm{O}}^{n}$ oxygen character of the DOS near the valence band maximum, using the same color key. 




Figure 6: 2D density plots of energy versus localization (IPR) are shown for each set of $\mathrm{Q}_{\mathrm{O}}^{n}$ states in the valence band, from Fig. 5 . The approximate position of the mobility edge, as determine in Fig. 5, is denoted by the dashed vertical lines. Note the different vertical scales of the top row versus the bottom row.

configurations for 30:70NS, plotted on top of the DOS. Recall that the IPR measures the degree of localization of each electronic state and ranges from 1 to $N$ (see Eq. 1), with 1 denoting a completely delocalized state and higher values characterizing increased localization. By projecting the charge density onto atomic orbitals and analyzing the partial DOS, we confirm that the top of the valence band is oxygen $2 p$ in character and the localized traps in the mobility gap are mostly associated with non-bridging oxygen atoms. The $\mathrm{Q}_{\mathrm{O}}^{n}$ character of a state is assigned by noting which of the $\mathrm{Q}_{\mathrm{O}}^{n}$ oxygen give the highest contribution to the partial density for each state, as described in Section 3.1, and is plotted in Fig. 5 using different colors for each $\mathrm{Q}_{\mathrm{O}}^{n}$.

In Fig. 5, a decomposition of the DOS based on $\mathrm{Q}_{\mathrm{O}}^{n}$ character is plotted below the energy axis, by building histograms of the $\mathrm{Q}_{\mathrm{O}}^{n}$-decomposed states close to the valence band maximum. (n.b. While similar to partial DOS, these histograms are normalized for clarity, rather than weighted by orbital or atom type.) From these histograms, the shoulder of the valence band is seen to be primarily of $\mathrm{Q}_{\mathrm{O}}^{1}$ and $\mathrm{Q}_{\mathrm{O}}^{2}$ character; calculations of the IPR show the states are highly localized. On the other hand, the main valence band peak is primarily of $\mathrm{Q}_{\mathrm{O}}^{4}$ and $\mathrm{Q}_{\mathrm{O}}^{3}$ character. Also note that the vast majority of 


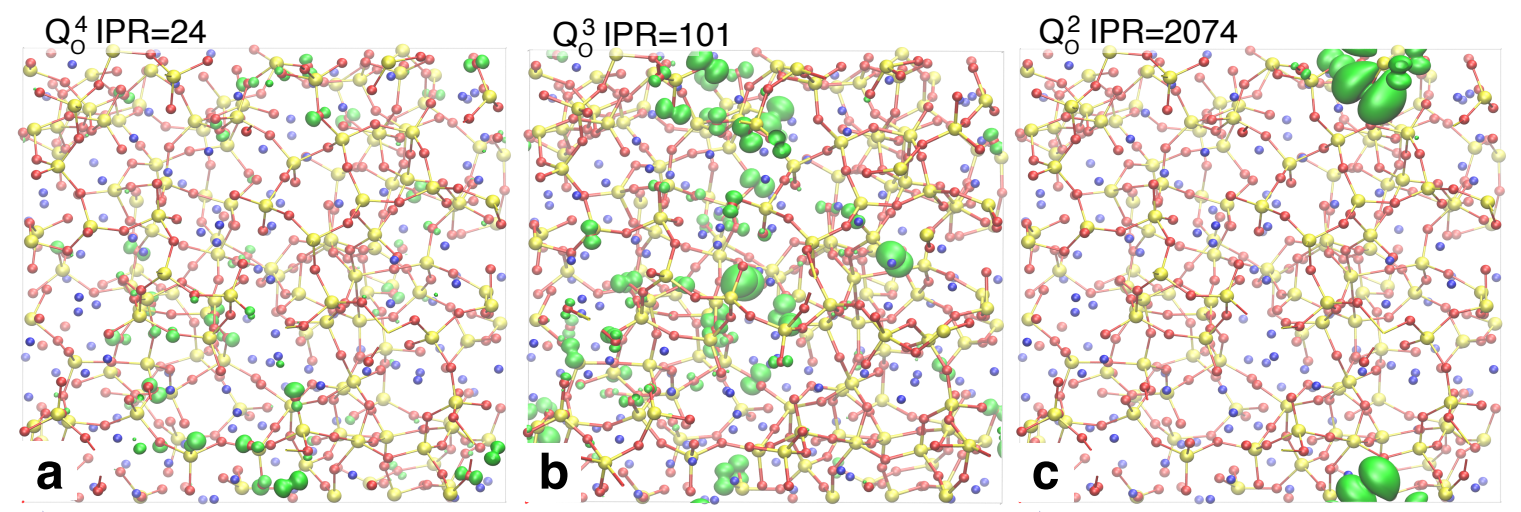

Figure 7: Representative partial charge densities for states with (a) delocalized character with $\log (\mathrm{IPR})=3.18$ (low IPR, $\left.\mathrm{Q}_{\mathrm{O}}^{4}\right)$, (b) intermediate character with $\log (\mathrm{IPR})=4.62$ ( $\sim$ threshold IPR, $\mathrm{Q}_{\mathrm{O}}^{3}$ ), and (c) localized character with $\log (\mathrm{IPR})=7.64$ (high IPR, $\mathrm{Q}_{\mathrm{O}}^{2}$ ). The same isosurface value is plotted in each frame.

$\mathrm{Q}_{\mathrm{O}}^{1}$ and $\mathrm{Q}_{\mathrm{O}}^{2}$ states have IPR values above the localization threshold defining the mobility edge, while many $\mathrm{Q}_{\mathrm{O}}^{3}$ and nearly all $\mathrm{Q}_{\mathrm{O}}^{4}$ states are below the delocalization threshold (conducting states).

The bottom of the conduction band in silica and sodium silicate is primarily derived from silicon $3 s$ orbitals (mixed with some oxygen $2 s$ and $2 p$ orbitals), which are more delocalized and less directional than the oxygen $2 p$ orbitals [29]. Thus, $\mathrm{Q}_{\mathrm{O}}^{n}$ values are not assigned to states in the conduction band, nor are the IPR values for conduction band states plotted in Fig. 5. Also, since in Fig. 5 many IPR values are obscured due to overlapping of states and the compressed logarithmic scale, density plots of the IPR values vs. energy on linear scales are shown for each $\mathrm{Q}_{\mathrm{O}}^{n}$ separately in Fig. 6. In Fig. 6, the much higher IPR values associated with $Q_{O}^{1}$ in particular and also $\mathrm{Q}_{\mathrm{O}}^{2}$ (note the different vertical scales in the figure) are apparent, as well as their deeper extent into the band tail region.

\section{Discussion}

Increased sodium content in sodium silicate glass leads to increased concentration of NBOs and in particular increased concentration of more defective silica tetrahedra with lower $\mathrm{Q}^{n}$ values. By correlating the charge localization of states near the band gap with local structural features characterized by $\mathrm{Q}_{\mathrm{O}}^{n}$, our results clearly show how these NBO create hole traps near the valence band edge. Such a decomposition of the electronic structure 
of an amorphous material by NBO type is a new analysis that also allows us to determine the mobility edge in the material.

In Fig. 6, the 2D density plots of the IPR versus energy for each state separated by $\mathrm{Q}_{\mathrm{O}}^{n}$ show that the $\mathrm{Q}_{\mathrm{O}}^{4}$-associated states are delocalized with a very narrow spread of their IPR values, all with $\log (\mathrm{IPR})$ values below 100 , and energies well below the highest occupied states (more than $2 \mathrm{eV}$ ). On the other hand, the states associated with $\mathrm{Q}_{\mathrm{O}}^{1}$ and $\mathrm{Q}_{\mathrm{O}}^{2}$ oxygen have a wide spread over many IPR values and extend into the band tail shoulder of the DOS. Qualitatively, as the $\mathrm{Q}_{\mathrm{O}}^{n}$ number decreases, the localization and energy of the trap state increases. Using the differences in energy and localization, the mobility gap can be estimated.

We estimate the mobility edge in the valence band is around $-1.8 \mathrm{eV}$ below the highest filled valence state for 30:70NS, as indicated in Figs. 5 and 6. States with energies higher than $-1.8 \mathrm{eV}$ do not have a majority $\mathrm{Q}_{\mathrm{O}}^{4}$ character and their localization begins to increase sharply compared to the delocalization of states with energies lower than $-1.8 \mathrm{eV}$. To guide the eye, a horizontal line is drawn at $\log (\mathrm{IPR})=3.65$ in Fig. 5 , to show that the $\log (\mathrm{IPR})$ of most of the delocalized $\mathrm{Q}_{\mathrm{O}}^{4}$ character states are lower than 3.65 while all of the states with energies higher than $-1.8 \mathrm{eV}$ have a $\log (\mathrm{IPR})$ larger than 3.65. Figure 7 shows isosurfaces of representative states below, near, and above this threshold to demonstrate their localization or delocalization; note again the typical association of higher $\mathrm{Q}_{\mathrm{O}}^{n}$ values with more delocalized states, although of course states with similar IPR values show similar delocalization regardless of $\mathrm{Q}_{\mathrm{O}}^{n}$.

The $\mathrm{Q}_{\mathrm{O}}^{1}$ and $\mathrm{Q}_{\mathrm{O}}^{2}$ states extend far into the mobility gap, creating both shallow and deep traps for hole carriers. These traps are inevitable in alkalisilicates, which gives a good explanation for why these network glasses do not scintillate compared to Ce-doped silica[10]. The $\mathrm{Q}_{\mathrm{O}}^{3}$ states are less localized than the $\mathrm{Q}_{\mathrm{O}}^{1}$ and $\mathrm{Q}_{\mathrm{O}}^{2}$ states (see Fig. 7b), with many states having low IPR values comparable to the $\mathrm{Q}_{\mathrm{O}}^{4}$ states, as is clearly seen in the bottom row of Fig. 6. A high concentration of such partially-localized states could form conducting channels by overlapping, however in the case of sodium silicate this always occurs with the introduction of a comparably large concentration of strongly-trapping $\mathrm{Q}_{\mathrm{O}}^{1}$ and $\mathrm{Q}_{\mathrm{O}}^{2}$ states, which inhibit hole transport. In addition, a significant fraction of $\mathrm{Q}_{\mathrm{O}}^{3}$ states are localized, with up to 2 orders of magnitude higher IPR than the bands with only tetrahedral $\mathrm{Q}_{\mathrm{O}}^{4}$ character. However, since some $\mathrm{Q}_{\mathrm{O}}^{3}$ states are present in pure silica, it is clear that these states do not completely quench the transport of holes, as scintillation has 
been observed in Ce-doped silica.

Our simulations quantify the nature of NBOs that trap hole carriers in sodium silicate compared to silica and explain the reason sodium silicate is not a good scintillator host, and in particular worse compared to pure silica. The concentration of the most detrimental hole traps increases with sodium concentration as additional NBOs further disrupt the tetrahedral silica network. In addition, our analysis suggests that the light yield from Cedoped silica could be increased if 1 ) the number of $\mathrm{Q}^{3}$ states could be reduced or 2) the density of Ce activators could be increased. A higher density of Ce dopants would require a shorter diffusion path for the carriers, giving less likelihood that they encounter a trap befor reaching an activator $(\mathrm{Ce})$ atom [7]. However, much previous research has been devoted to removing dangling bonds in silica via control of the glass processing procedure $[30,10,37]$, so it is unlikely that the remaining (mostly $\mathrm{Q}^{3}$ ) hole traps can be removed without modification of the glass composition. Furthermore, the applicability of direct atomistic simulations of the glass manufacturing processes to search for such opportunities is probably limited, since the relevant timescales are much longer than accessible even with classical potentials.

\section{Conclusions}

In summary, we calculated the electronic structure of 30:70 sodium silicate glass using density functional theory on an ensemble of 30 configurations generated with classical potentials. We provide a more detailed analysis of the localization and character of states near the band edges than has been previously reported for alkali silicates, with a direct correlation shown between local atomic and electronic structure. Our analysis reveals that non-bridging oxygens introduced by the alkali ions can create many highly localized hole traps at the top of the valence band. In particular, the NBO from $\mathrm{Q}^{1}$ and $\mathrm{Q}^{2}$ states extend far into the mobility gap and are significantly more localized than $\mathrm{Q}^{3}$ NBOs. Any attempt to increase conductivity by introducing network-disrupting alkali ions to create overlapping shallow traps inevitably produces a concomitantly large concentration of deep traps that inhibit hole transport.

\section{Acknowledgement}

This work was performed under the auspices of the U.S. Department of Energy by Lawrence Livermore National Laboratory under Contract DE- 
Table A.2: The potential parameters for $\mathrm{Si}, \mathrm{O}$, and $\mathrm{Na}$, where $\mathrm{p}=0.26 \AA$ for every pair.

\begin{tabular}{lccccc}
$i j k$ & $\mathrm{~A}_{i j}$ & $1 / \beta_{i j}$ & $\lambda_{i j k}$ & $\gamma_{i j k}$ & $r_{i}^{c}$ \\
\hline $\mathrm{Si}-\mathrm{Si}$ & $1171.53 \mathrm{eV}$ & $0.43471 / \AA$ & $149.796 \mathrm{eV}(\mathrm{i}=\mathrm{Si})$ & $2.6 \AA(\mathrm{i}=\mathrm{Si})$ & 2.6 \\
$\mathrm{Si}-\mathrm{O}$ & $1848.73 \mathrm{eV}$ & $0.42741 / \AA$ & & & \\
$\mathrm{Si}-\mathrm{Na}$ & $1248.92 \mathrm{eV}$ & $0.43481 / \AA$ & & & \\
$\mathrm{Na}-\mathrm{Na}$ & $1347.54 \mathrm{eV}$ & $0.45451 / \AA$ & $0(\mathrm{i}=\mathrm{Na})$ & $0(\mathrm{i}=\mathrm{Na})$ & 0 \\
$\mathrm{Na}-\mathrm{O}$ & $1994.16 \mathrm{eV}$ & $0.42741 / \AA$ & & & \\
$\mathrm{O}-\mathrm{O}$ & $452.509 \mathrm{eV}$ & $0.42741 / \AA$ & & & \\
$\mathrm{O}-\mathrm{Si}-\mathrm{Si}$ & & & $6.2415 \mathrm{eV}$ & $2.0000 \AA$ & $3.00 \AA$ \\
O-Si-Na & & & $6.2415 \mathrm{eV}$ & $2.0021 \AA$ & $3.1 \AA$ \\
O-Na-Na & & & $6.2415 \mathrm{eV}$ & $2.0000 \AA$ & $3.2 \AA$
\end{tabular}

AC52-07NA27344 and funded by the DOE/NNSA Office of Defense Nuclear Nonproliferation Research and Development PDP WMS Team.

[1] J. C. Slater, G. F. Koster, Simplified LCAO method for the periodic potential problem, Physical Review 94 (6) (1954) 1498-1524.

[2] J. H. Los, T. D. Kühne, S. Gabardi, M. Bernasconi, First principles simulation of amorphous InSb, Physical Review B 87 (18) (2013) 184201.

[3] T. Mueller, E. Johlin, J. C. Grossman, Origins of hole traps in hydrogenated nanocrystalline and amorphous silicon revealed through machine learning, Physical Review B 89 (11) (2014) 115202.

[4] E. Auffray, N. Akchurin, A. Benaglia, A. Borisevich, C. Cowden, J. Damgov, V. Dormenev, C. Dragoiu, P. Dudero, M. Korjik, D. Kozlov, S. Kunori, P. Lecoq, S. W. Lee, M. Lucchini, V. Mechinsky, K. Pauwels, DSB:Ce 3+scintillation glass for future, Journal of Physics: Conference Series 587 (2015) 012062. 
[5] Z. H. Stachurski, On structure and properties of amorphous materials, Materials 4 (9) (2011) 1564-1598.

[6] I. Avramov, T. Vassilev, I. Penkov, The glass transition temperature of silicate and borate glasses, Journal of Non-Crystalline Solids 351 (6-7) (2005) 472-476.

[7] W. Chewpraditkul, Y. Shen, D. Chen, B. Yu, P. Prusa, M. Nikl, A. Beitlerova, C. Wanarak, Luminescence and scintillation of Ce3+doped high silica glass, Optical Materials 34 (11) (2012) 1762-1766.

[8] N. Mott, Electrons in glass, Contemporary Physics 18 (3) (1977) 225245.

[9] A. N. Trukhin, M. N. Tolstoi, L. B. Glebov, Elementary electronic excitations in pure sodium silicate glasses, physica status solidi (b) 99 (155) (1980) 155-162.

[10] M. Bliss, R. A. Craig, P. L. Reeder, The physics and structure-property relationships of scintillator materials: effect of thermal history and chemistry on the light output of scintillating glasses, Nuclear Instruments and Methods in Physics Research Section A: Accelerators, Spectrometers, Detectors and Associated Equipment 342 (2) (1994) 357-363.

[11] M. Bliss, P. M. Aker, C. F. Windisch, Jr, Further investigations of the effect of replacing lithium by sodium on lithium silicate scintillating glass efficiency, Journal of Non-Crystalline Solids 358 (4) (2012) 751-757.

[12] M. Nikl, Energy transfer phenomena in the luminescence of wide bandgap scintillators, physica status solidi (a) 202 (2) (2005) 201-206.

[13] J. Dong, D. A. Drabold, Atomistic structure of band-tail states in amorphous silicon, Physical Review Letters 80 (9) (1998) 1928.

[14] D. A. Drabold, U. Stephan, J. Dong, S. M. Nakhmanson, The structure of electronic states in amorphous silicon, Journal of Molecular Graphics and Modelling 17 (1999) 330-332. 
[15] S. Ispas, M. Benoit, P. Jund, R. Jullien, Structural and electronic properties of the sodium tetrasilicate glass Na2Si4O9 from classical and ab initio molecular dynamics simulations, Physical Review B 64 (21) (2001) 214206.

[16] A. Tilocca, N. H. de Leeuw, Structural and electronic properties of modified sodium and soda-lime silicate glasses by Car?Parrinello molecular dynamics, Journal of Materials Chemistry 16 (20) (2006) 1950.

[17] R. Biswas, G. S. Grest, C. M. Soukoulis, Generation of amorphoussilicon structures with use of molecular-dynamics simulations, Physical Review B 36 (14) (1987) 7437.

[18] H. Melman, S. H. Garofalini, Microstructural evaluation of simulated sodium silicate glasses, Journal of Non-Crystalline Solids 134 (1) (1991) $107-115$.

[19] S. Plimpton, Fast parallel algorithms for short-range molecular dynamics, Journal of Computational Physics 117 (1) (1995) 1-19.

[20] J. P. Perdew, K. Burke, M. Ernzerhof, Generalized gradient approximation made simple, Phys. Rev. Lett. 77 (18) (1996) 3865-3868. doi:10.1103/PhysRevLett.77.3865.

[21] J. P. Perdew, K. Burke, M. Ernzerhof, Erratum: Generalized gradient approximation made simple, Phys. Rev. Lett. 78 (1997) 1396.

[22] G. Kresse, J. Hafner, Ab initio molecular dynamics for liquid metals, Physical Review B 47 (1) (1993) 558-561.

[23] G. Kresse, J. Hafner, Ab initio molecular-dynamics simulation of the liquid-metal-amorphous-semiconductor transition in germanium, Physical Review B 49 (20) (1994) 14251.

[24] G. Kresse, J. Furthmüller, Efficient iterative schemes for ab initio totalenergy calculations using a plane-wave basis set, Physical Review B 54 (16) (1996) 11169.

[25] G. Kresse, J. Furthmüller, Efficiency of ab-initio total energy calculations for metals and semiconductors using a plane-wave basis set, Comput. Mater. Sci. 6 (1) (1996) 15-50. 
[26] P. E. Blöchl, Projector augmented-wave method, Phys. Rev. B 50 (24) (1994) 17953-17979. doi:10.1103/PhysRevB.50.17953.

[27] G. Kresse, D. Joubert, From ultrasoft pseudopotentials to the projector augmented-wave method, Phys. Rev. B 59 (3) (1999) 1758-1775. doi:10.1103/PhysRevB.59.1758.

[28] B. Sadigh, P. Erhart, D. Åberg, A. Trave, E. Schwegler, J. Bude, FirstPrinciples Calculations of the Urbach Tail in the Optical Absorption Spectra of Silica Glass, Physical Review Letters 106 (2) (2011) 027401.

[29] S. Hosokawa, H. Sato, K. Mimura, Y. Tezuka, D. Fukunaga, Y. Matsuzaki, F. Shimojo, Oxygen 2 pPartial Density of States and Bond Angles around O Atoms in SiO 2Glass, Journal of the Physical Society of Japan 84 (2) (2015) 024605.

[30] J. F. Stebbins, Effects of temperature and composition on silicate glass structure and dynamics: Si-29 NMR results, Journal of Non-Crystalline Solids 106 (1) (1988) 359-369.

[31] H. Maekawa, T. Maekawa, K. Kawamura, T. Yokokawa, The structural groups of alkali silicate glasses determined from 29 Si MAS-NMR, Journal of Non-Crystalline Solids 127 (1) (1991) 53-64.

[32] M. Pota, A. Pedone, G. Malavasi, C. Durante, M. Cocchi, M. C. Menziani, Molecular dynamics simulations of sodium silicate glasses: Optimization and limits of the computational procedure, Computational Materials Science 47 (3) (2010) 739-751.

[33] J. Du, A. N. Cormack, The medium range structure of sodium silicate glasses: a molecular dynamics simulation, Journal of Non-Crystalline Solids 349 (2004) 66-79.

[34] J. Du, A. N. Cormack, The structure of erbium doped sodium silicate glasses, Journal of Non-Crystalline Solids 351 (27-29) (2005) 2263-2276. 
[35] A. C. Wright, The comparison of molecular dynamics simulations with diffraction experiments, Journal of Non-Crystalline Solids 159 (3) (1993) 264-268.

[36] X. Yuan, A. N. Cormack, Local structures of MD-modeled vitreous silica and sodium silicate glasses, Journal of Non-Crystalline Solids 283 (1) (2001) 69-87.

[37] B. M. Lee, H. K. Baik, B. S. Seong, S. Munetoh, T. Motooka, Generation of glass $\mathrm{SiO} 2$ structures by various cooling rates: A molecular-dynamics study, Computational Materials Science 37 (3) (2006) 203-208. 